\title{
Article \\ Chemoresistive Sensor Readout Circuit Design for Detecting Gases with Slow Response Time Characteristics
}

\author{
Dong-Yeon Lee, Joon-Boo Yu, Hyung-Gi Byun *(D) and Hyeon-June Kim * \\ Department of Electronics Engineering, Kangwon National University, Samcheok 25913, Korea; \\ ldylee5423@kangwon.ac.kr (D.-Y.L.); joonbou.yu@kangwon.ac.kr (J.-B.Y.) \\ * Correspondence: byun@kangwon.ac.kr (H.-G.B.); hyeonjunekkim@kangwon.ac.kr (H.-J.K.); \\ Tel.: +82-33-570-6401 (H.-G.B.); +82-33-570-6536 (H.-J.K.)
}

check for updates

Citation: Lee, D.-Y.; Yu, J.-B.; Byun, H.-G.; Kim, H.-J. Chemoresistive Sensor Readout Circuit Design for Detecting Gases with Slow Response Time Characteristics. Sensors 2022, 22, 1102. https://doi.org/10.3390/ s22031102

Academic Editor: Faisal Mohd-Yasin

Received: 29 December 2021

Accepted: 29 January 2022

Published: 31 January 2022

Publisher's Note: MDPI stays neutral with regard to jurisdictional claims in published maps and institutional affiliations.

Copyright: (C) 2022 by the authors. Licensee MDPI, Basel, Switzerland. This article is an open access article distributed under the terms and conditions of the Creative Commons Attribution (CC BY) license (https:// creativecommons.org/licenses/by/ $4.0 /)$.

\begin{abstract}
Based on an analysis of the signal characteristics of gas sensors, this work presents a chemoresistive sensor readout circuit design for detecting gases with slow response time characteristics. The proposed readout circuit directly generates a reference voltage corresponding to the initial value of the gas sensor and extracts only the amount of gas concentration change in the sensor. Because the proposed readout circuit can adaptively regenerate the suitable reference voltage under various changing ambient conditions, it can alleviate the variation in output values at the same gas concentration caused by non-uniformities among gas sensors. Furthermore, this readout circuit effectively eliminates the initial value shifts due to the poor reproducibility of the gas sensor itself without requiring complex digital signal calibrations. This work focuses on a commercially viable readout circuit structure that can effectively obtain slow response gas information without requiring a large capacitor. The proposed readout circuit operation was verified by simulations using spectre in cadence simulation software. It was then implemented on a printed circuit board with discrete components to confirm the effectiveness with existing gas sensor systems and its commercial viability.
\end{abstract}

Keywords: gas sensor; chemoresistive sensor; slow response gas sensing; readout circuit; offset tracking readout

\section{Introduction}

Recently, with the development of electronic devices, the demand for microsensor applications has been increased for improving the quality of human life. In particular, gas sensors such as electronic noses (e-noses) are being widely used in various electronic application fields, such as air-quality monitoring systems, hazardous gas detection systems, and healthcare systems [1-4]. To date, various types of gas sensors have been developed based on different sensing materials and their specialized sensing mechanisms [5-7]. Recently, metal-oxide-semiconductor (MOS)-based gas sensors are attracting attention with the development of semiconductor manufacturing technology owing to their compatibility with Internet of Things (IoT) applications. In addition, these sensors have chemoresistive characteristics and provide a simple interface circuitry for the signal conversion.

When a gas sensor is exposed to the target ambient conditions, depending on the sensing materials, their chemoresistance exhibits various ohmic distributions and different sensitivity and selectivity. The purpose of e-nose is to detect the type of target gas using the various sensing materials. From a signal and system's perspective, if the type of target gas can be clearly defined, the sensor interfaces and readout integrated circuits (ROICs) can be efficiently designed for the optimal signal extraction. However, due to inherent properties of the materials constituting the gas sensor (i.e., poor selectivity of gas sensors $[8,9])$, it is difficult to rely on the selectivity of gas sensors that should react only to a specific gas component. Moreover, the chemoresistive characteristics of gas sensors change in accordance with the surrounding conditions in the environment, such as 
temperature, humidity, and pressure [10]. This leads to different initial values of the gas sensor (i.e., offset variations) for each set of sensing measurements, causing non-uniformity of signal conversion results even with the same gas concentration. To alleviate this problem, because there is a limitation for improving gas sensor performance only through the physical structure and material modifications, the studies on gas sensing ROICs should be performed together with the sensor characteristics to effectively overcome the limits of gas sensor performances in this field.

As a conventional readout topology, most commercial chemoresistive gas sensors are based on a voltage-divider structure [11] for resistance-to-voltage conversion because it provides a wide dynamic range of the sensor resistance. In addition, to remove the initial value of gas sensors (i.e., gas sensor offsets), a differential amplifier and Wheatstone bridge circuit [12-14] are utilized to obtain only the amount of change of the gas sensor output with a fixed proper reference voltage. However, since the gas sensor has an offset variation for each sample, gas sensor offset cancelling circuits with a fixed reference voltage do not accurately extract the amount of change of the gas sensor output, resulting in a degradation of uniformity and dynamic range. This phenomenon is further exacerbated when the gas sensor offset is changed by the surrounding conditions in the environment.

Until now, various types of sensing methods have been reported: the oscillator-based sensing method [15] to perform low-power conversion, the digital-to-analog converter (DAC)-based method [16] to support wide-range resistance change, and the reconfigurable multiple sensing method [17] to detect various kinds of gases with different sensitivity and resistance characteristics. With the same motivation as previous works $[18,19]$, based on the analysis of the electrical signals of the gas sensor, this paper presents a slow-response gassensing readout circuit that minimizes the effect on sensor offset variation caused by various ambient conditions. After reading out a gas sensor offset, using an additional digital to analog convertor (DAC), the proposed readout circuit generates a suitable reference voltage for a differential amplifier. Then, while maintaining the uniformity for each gas sensor, the differential amplifier extracts only the amount of change in the gas sensor. Furthermore, because the proposed readout circuit adaptively generates the suitable reference voltage, the shifting effect of the sensor offset due to the reproducibility lack of gas sensors (i.e., drift effect) is effectively eliminated without complex digital signal calibrations. In this work, the proposed readout circuit operation and its performances were verified in by simulation using spectre in cadence simulation software. It was implemented on a printed circuit board (PCB) with discrete components to confirm its effectiveness with existing gas sensor systems and commercial viability.

The rest of this paper is organized as follows: Section 2 analyzes the characteristics of the gas sensor signals by power spectral density measurements. Section 3 describes the proposed readout circuit structure with the offset tracking technique. The measurement results and discussions about the proposed readout circuit are presented in Section 4, followed by the conclusion in Section 5 .

\section{Analysis of Output Characteristics of Sensor}

In this section, we focus on the signal characterization of the slow responsive gas sensor and derive an optimal sensing method, which can be directly applied to existing commercial gas sensors.

Figure 1a shows the conventional resistive-divider sensing structure with an ACcoupling capacitor $\left(C_{S}\right)$. $R_{S}$ represents the ohmic characteristic of the gas sensor and $R_{L}$ represents the load resistor to define the bias current. In commercial products, this structure is mostly utilized as the basic gas sensor readout circuit for resistance-to-voltage (RV) conversion, which permits only the amount of change in the gas sensor to be transmitted to the readout circuit through the $C_{S}$. Note that the gas sensor has a different offset value for each sample. Based on the published data sheet information for the Figaro TGS2600 [20,21] sensor, in the case of slow response gas sensing, it is assumed that the $R_{S}$ is varied from 100 to $1 \mathrm{k} \Omega$ depending on gas concentration and that the $R_{\mathrm{L}}$ is $100 \mathrm{k} \Omega$ under a supply voltage 
$\left(\mathrm{V}_{\mathrm{DD}}\right)$ of $3.3 \mathrm{~V}$. Figure $1 \mathrm{~b}$ shows the power spectral density (PSD) of the gas sensor outputs $\left(\mathrm{V}_{\mathrm{SO}}\right.$ and $\mathrm{V}_{\mathrm{IN}}$ ) according to the capacitance of $\mathrm{C}_{\mathrm{S}}$. For the condition of the same sensing time (equal to settling time of the $R_{S}$ ), the signal power of the $V_{I N}$ varies according to the capacitance of $C_{S}$. Note that $V_{I N}$ is the input signal to the gas sensor that is actually read out by the next stage readout circuit. It is clear from the PSD results that the low frequency signal power is dominant in the total output signal power of the gas sensors, and that a large $C_{S}$ is needed to extract the amount of change. When the capacitance of $C_{S}$ becomes larger than $1 \mathrm{nF}$, the PSDs of the $\mathrm{V}_{\mathrm{SO}}$ and $\mathrm{V}_{\mathrm{IN}}$ become similar.

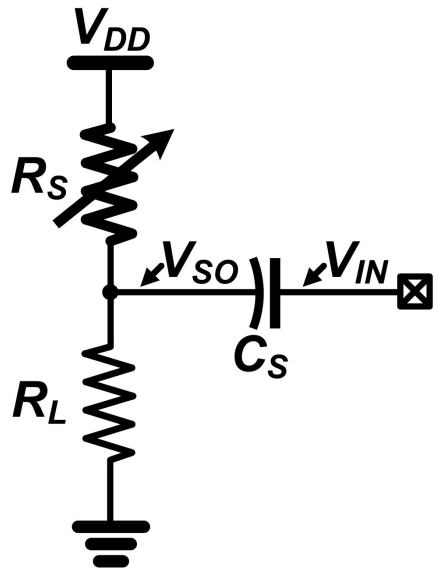

(a)

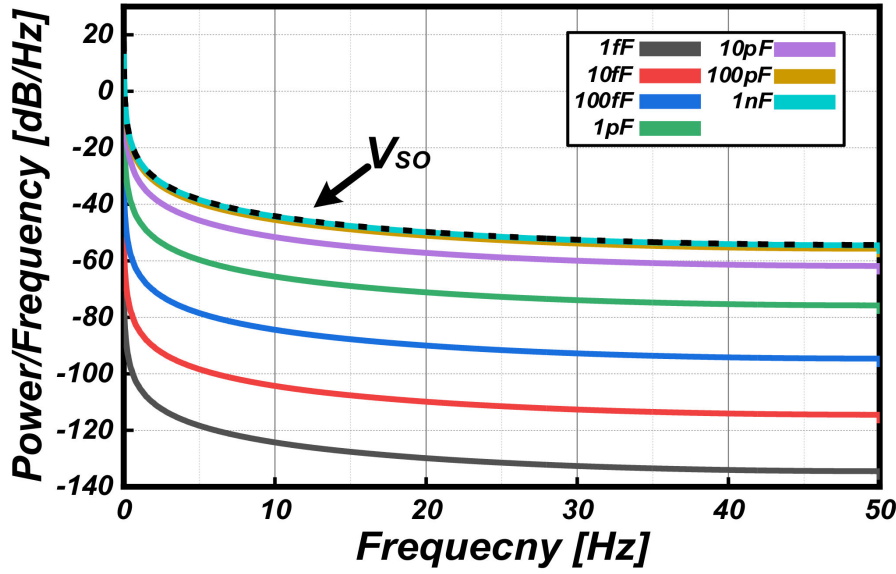

(b)

Figure 1. (a) Conventional resistive-divider sensing structure with an AC-coupling capacitor. (b) Power spectral density of the gas sensor outputs according to the capacitance of $\mathrm{C}_{\mathrm{S}}$.

To further investigate the signal characteristics for the condition of the same gas concentration, the PSDs of $\mathrm{V}_{\mathrm{IN}}$ was analyzed for each sensitivity of the gas sensor. The sensitivity of each gas sensor is modeled with different settling times of $R_{S}$ for the same sensing time. Here, the settling time of $R_{S}$ from 1 to $10,000 \mathrm{~s}$ was considered with a $C_{S}$ of $1 \mathrm{pF}$. Figure 2a shows the PSD results of $\mathrm{V}_{\mathrm{SO}}$ according to changes in the settling time of $\mathrm{R}_{\mathrm{S}}$. With a constant capacitance of $\mathrm{C}_{\mathrm{S}}$, which is to be expected, the proportion of signal power for each frequency decreases as the required settling time for $R_{S}$ increases. This implies that a suitable capacitance of $\mathrm{C}_{\mathrm{S}}$ should be considered for accurate signal transmission, depending on the sensitivity of the target gas sensor. In Figure $2 b$, for obtaining the signal transmission over than 3-sigma $(3 \delta)$ within the sensing time of $1 \mathrm{~s}$, the required capacitance of $\mathrm{C}_{\mathrm{S}}$ is shown according to the settling time of $\mathrm{R}_{\mathrm{S}}$. Note that the sensitivity of each sensor was modeled in proportion to the settling time. When the settling time of $R_{S}$ is relatively decreased by 10 times, the size of $\mathrm{C}_{\mathrm{S}}$ should be increased approximately 10 times to readout the amount of change of the $R_{S}$ with $3 \delta$ accuracy. This indicates that the size of $C_{S}$ should be increased when using a gas sensor with a low sensitivity and slow response characteristics. However, even with the optimized capacitance $C_{S}$ for each gas sensor, the sensing results might be different each time due to the variation of the sensitivity and reactivity according to changes in the ambient conditions.

In term of commercialization, the increasing capacitor size is disadvantageous in price competitiveness. Moreover, it is difficult to utilize different capacitance sizes that depend on the target ambient conditions. For all these reasons, it is worthwhile to study the slow-response gas-sensing readout circuit structure without using a large size capacitance to eliminate the gas sensor offset. 


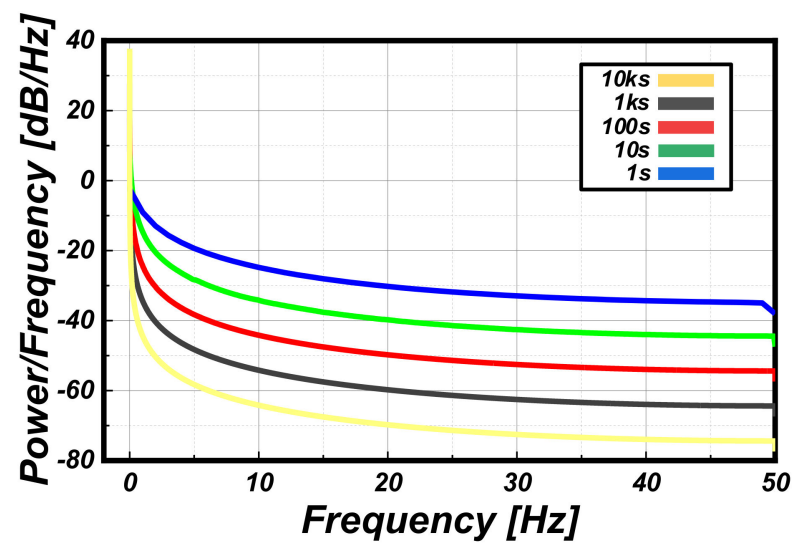

(a)

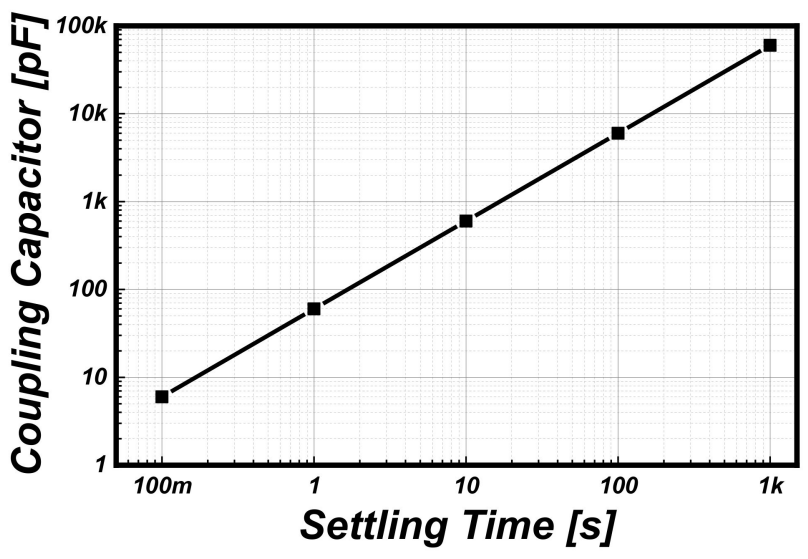

(b)

Figure 2. (a) PSD results of $\mathrm{V}_{\mathrm{SO}}$ according to changes in the settling time of $\mathrm{R}_{\mathrm{S}}$ and (b) the capacitance required for $\mathrm{V}_{\mathrm{IN}}$ settling according to the gas sensor's sensitivity.

\section{Proposed Readout Circuit Structure and Its Operation}

As discussed in the previous section, for gas sensors that respond slowly to ambient target gases, it is inefficient to apply the correlated-double-sampling (CDS) techniques as in $[18,19]$, which utilize an AC-coupling capacitor in readout circuit input networks to remove the gas sensor offset. In general, commercial gas sensors have different requirements for their optimal performances, which are not optimized for a specific readout circuit configuration and operation. Thus, we focus on a commercially viable readout circuit structure that can effectively obtain the measured information of gases with low reactivity and sensitivity characteristics, while maintaining compatibility with the gas sensor itself.

Figure 3 presents the proposed readout circuit structure for alleviating the sensor offset variation, which consists of a differential amplifier, a 14-bit analog-to-digital converter (ADC), and a 14-bit DAC. Compared to the conventional readout circuit, instead of an additional reference-generating circuit [12-14], the reference-generating DAC has two readout paths (sensor offset readout and feedback). To verify the performance according to the change of the gas sensor offset, the gas sensor was modeled by dividing it into the sensing part $\left(\Delta R_{S}\right)$ and the offset part $\left(R_{O S}\right)$. In addition, to obtain accurate simulation results, the real signal waveforms measured by the Figaro TGS2600 sensor were used as $\Delta \mathrm{R}_{\mathrm{S}}$. Matching actual design specifications, the differential amplifier was designed with an open loop gain of $95 \mathrm{~dB}$, a common mode rejection rate of $85 \mathrm{~dB}$, a power supply rejection rate of $80 \mathrm{~dB}$, and a slew rate of $160 \mathrm{MHz}$. Here, $R_{\mathrm{L}}, \mathrm{R}_{1}$, and $\mathrm{R}_{2}$ were set as $\sim 10 \mathrm{k} \Omega, \sim 50 \mathrm{k} \Omega$, and $\sim 50 \mathrm{k} \Omega$, respectively. For resetting the gas sensor, the supply voltage $\left(\mathrm{V}_{\mathrm{DD}}\right)$ for the voltage-driving of the gas sensor can be changed to the ground voltage $\left(\mathrm{V}_{\mathrm{SS}}\right)$.

The functional timing diagram of the proposed readout circuit is illustrated in Figure 4, with three phases of offset tracking $\left(\mathrm{T}_{\mathrm{OT}}\right)$, signal readout $\left(\mathrm{T}_{\mathrm{SR}}\right)$, and sensor reset $\left(\mathrm{T}_{\mathrm{RST}}\right)$. First, the offset tracking is performed during $\mathrm{T}_{\mathrm{OT}}$, to define the output common voltage of $V_{S O}$. After $\varnothing_{O S}$ is on, the ADC starts to read $V_{O S}$ during $\varnothing_{S A}$, and its results ( $D_{\text {INIT }}$ ) are transferred to the DAC. Then, when $\varnothing_{\mathrm{SD}}$ is on, the DAC regenerates $V_{\mathrm{REF}}$ corresponding to the $D_{\text {INIT, }}$ which is maintained until just before $T_{R S T}$. During $T_{S R}$, the differential amplifier performs the operation of subtracting $\mathrm{V}_{\mathrm{REF}}$ from $\mathrm{V}_{\mathrm{SO}}$, and the $\mathrm{ADC}$ converts it into digital codes ( $\left.D_{\text {SIG }}\right)$ with $\varnothing_{\text {RA }}$ and $\varnothing_{\text {SA }}$. The output result is only the amount of change of $V_{\text {SO }}$. Finally, during $\mathrm{T}_{\mathrm{RST}}$, the reset operation is performed to initialize the gas sensor itself. In this way, the gas sensor offset can be updated periodically, and even non-periodically, when replacing the gas sensor.

The simulation results of the conventional and proposed readout circuit are shown in Figures 5 and 6 with the real signal waveforms in the two cases: the case where the sensor offset changes upwards (Figure 5a) and the case where the sensor offset changes downwards (Figure 6a). Using the Figaro TGS2600 sensor, the gas-sensing results were 
obtained four times with alternately repeating injection of ethanol $\left(\mathrm{C}_{2} \mathrm{H}_{5} \mathrm{OH}\right)$ and fresh air. The different gas sensor offsets according to ambient condition changes were generated by replacing the sensor samples each sensing time. Table 1 shows the information about the real signal waveforms with the mean sensor resistance $R_{S}\left(=\Delta R_{S}+R_{O S}\right)$. The resistance value of the gas sensor was estimated by the equation of $\mathrm{R}_{\mathrm{S}} \approx\left(\left(\mathrm{V}_{\mathrm{DD}}-\mathrm{V}_{\mathrm{SO}}\right) / \mathrm{V}_{\mathrm{SO}}\right) \times \mathrm{R}_{\mathrm{L}}$. For the conventional readout circuit (representing in Figure $5 \mathrm{~b}$ ), whole gas-sensing periods from 1st to 4th, only the offset value of the first gas sensor is used as $V_{\text {REF }}$ to remove the gas sensor offset; the offsets $(0.65 \mathrm{~V}$ for the 2 nd offset change, $0.572 \mathrm{~V}$ for the 3rd offset change, and $0.436 \mathrm{~V}$ for the 4 th offset change) are larger than the first defined $\mathrm{V}_{\text {REF }}$ of $0.284 \mathrm{~V}$ (from the 1st sensor offset). Because the conventional readout circuit extracts just difference between $\mathrm{V}_{\mathrm{SO}}$ and $\mathrm{V}_{\mathrm{REF}}$, the offset variation is not properly removed (offset error) and rather is amplified as the output signal, which exacerbates the non-uniformity in measuring the same gas concentration. On the other hand, as shown in Figure 6b, when the sensor offsets $(0.594 \mathrm{~V}$ for 2 nd offset change, $0.443 \mathrm{~V}$ for 3rd offset change, and $0.692 \mathrm{~V}$ for 4 th offset change) are lower than the first defined $V_{\text {REF }}$ of $0.706 \mathrm{~V}$ (from 1st sensor offset), the output signal is clamped by the offset error, resulting in degradation of the sensing accuracy. These errors are because the conventional readout circuit does not reflect the change in the initial value of the input signal at all.

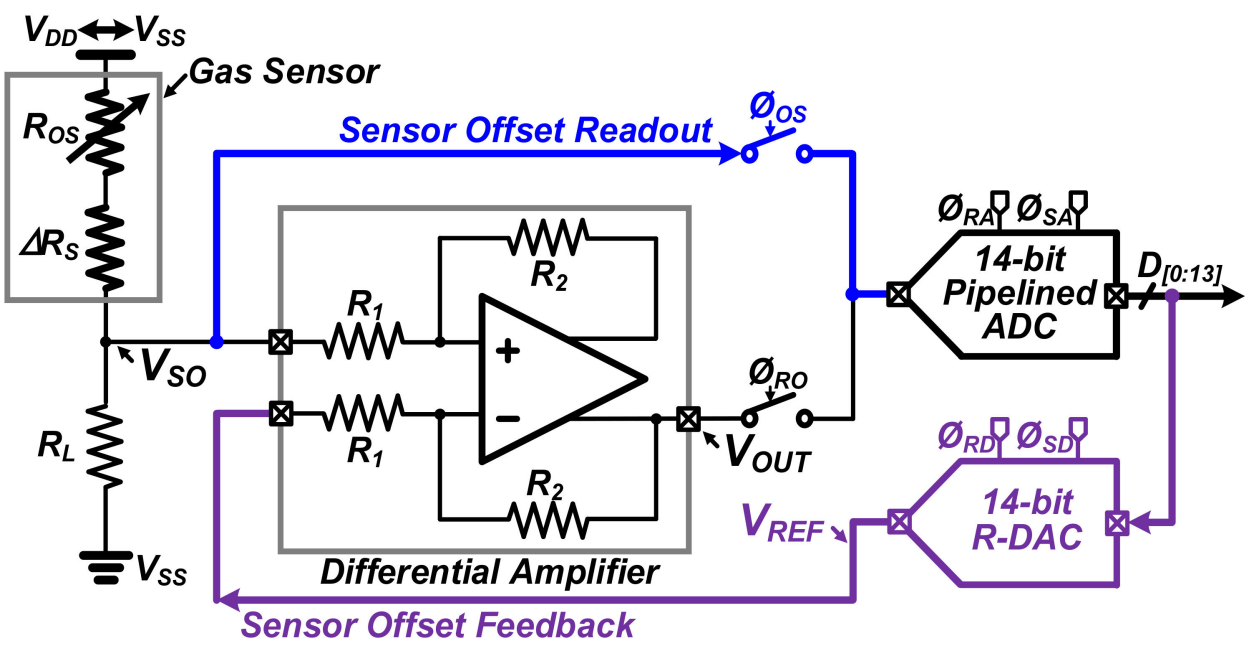

Figure 3. Proposed readout circuit structure for alleviating the sensor offset variation.

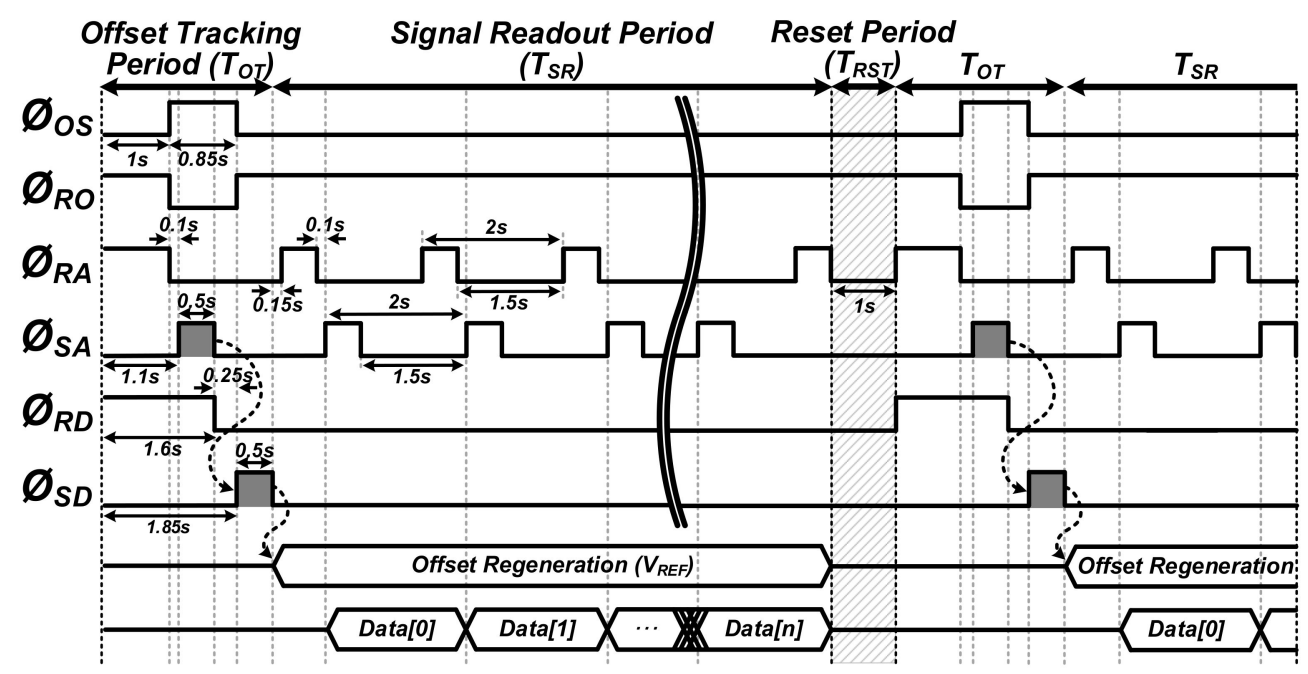

Figure 4. Operational timing diagram of the proposed readout circuit with three phases of offset tracking $\left(\mathrm{T}_{\mathrm{OT}}\right)$, signal readout $\left(\mathrm{T}_{\mathrm{SR}}\right)$, and sensor reset $\left(\mathrm{T}_{\mathrm{RST}}\right)$. 


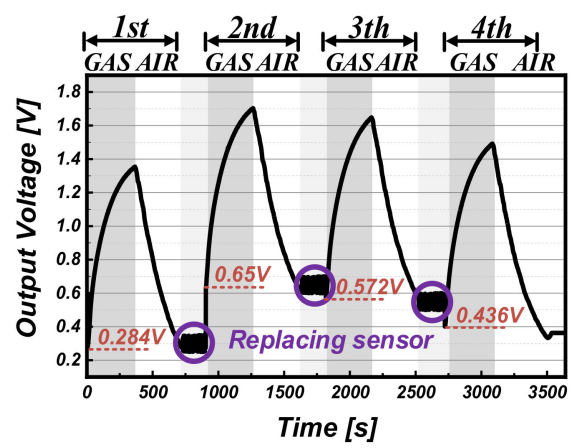

(a)

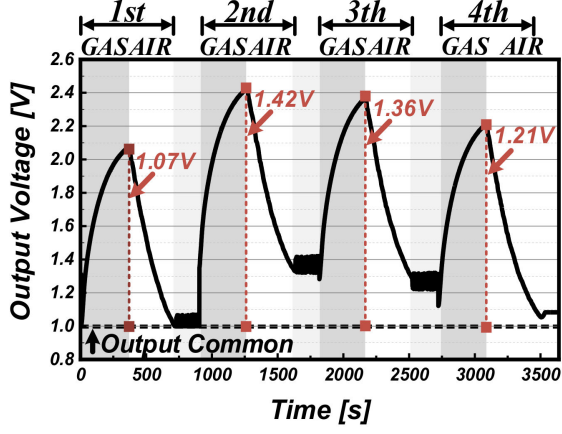

(b)

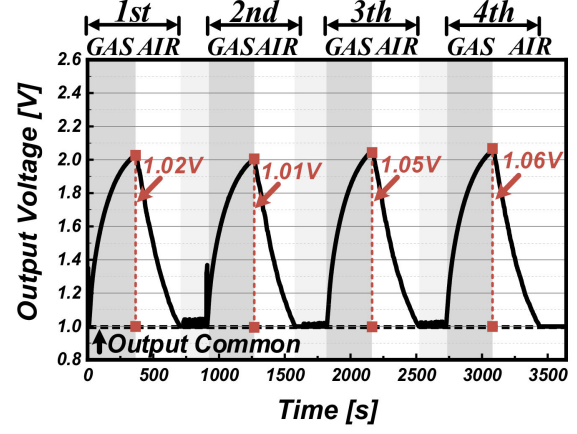

(c)

Figure 5. (a) Real signal waveform with changes in the sensor offset upwards; simulation results of the (b) conventional readout circuit and the (c) proposed readout circuit for that input.

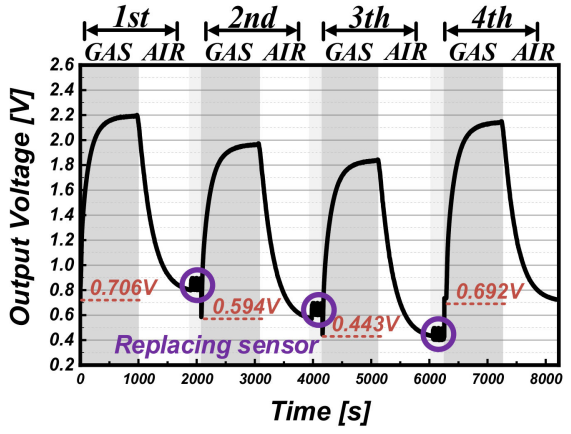

(a)

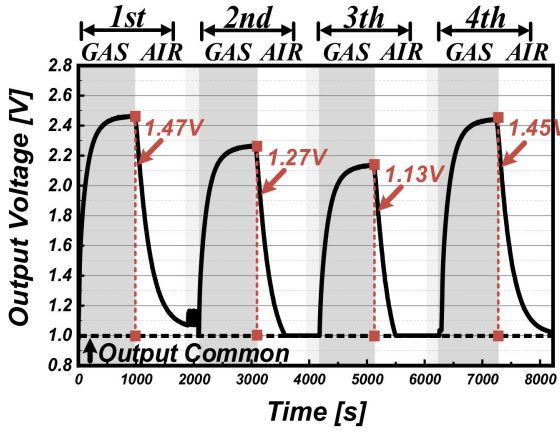

(b)

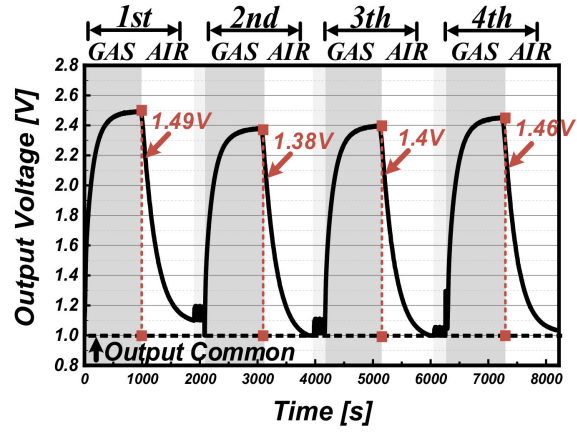

(c)

Figure 6. (a) Real signal waveform with changes in the sensor offset downwards; simulation results of the (b) conventional readout circuit and the (c) proposed readout circuit for that input.

Table 1. Information regarding the real signal waveforms with the mean sensor resistance.

\begin{tabular}{|c|c|c|c|c|c|}
\hline \multirow[b]{2}{*}{ Input Condition } & \multirow{2}{*}{$\begin{array}{c}\text { Ethanol } \\
\text { Concentration } \\
{[p p m]}\end{array}$} & \multicolumn{2}{|c|}{ Figure $5 \mathbf{a}$} & \multicolumn{2}{|c|}{ Figure $6 a$} \\
\hline & & $\begin{array}{l}\text { Measurement } \\
\text { Time [s] }\end{array}$ & $\begin{array}{c}\text { Mean Sensor } \\
\text { Resistance }[\mathrm{k} \Omega]\end{array}$ & $\begin{array}{l}\text { Measurement } \\
\text { Time [s] }\end{array}$ & $\begin{array}{c}\text { Mean Sensor } \\
\text { Resistance }[\mathrm{k} \Omega]\end{array}$ \\
\hline Ethanol & 50 & $0 \sim 360$ & $166.06 \sim 26.93$ & $0 \sim 950$ & $60.82 \sim 12.83$ \\
\hline Air & 0 & $360 \sim 720$ & $26.93 \sim 154.47$ & $950 \sim 1900$ & $12.83 \sim 50.98$ \\
\hline Replacing Sensor & 0 & $720 \sim 900$ & $x$ & $1900 \sim 2080$ & $x$ \\
\hline Ethanol & 50 & $900 \sim 1260$ & $62.46 \sim 19.43$ & $2080 \sim 3030$ & $74.18 \sim 15.35$ \\
\hline Air & 0 & $1260 \sim 1620$ & $19.43 \sim 70.39$ & $3030 \sim 3980$ & $15.35 \sim 77.57$ \\
\hline Replacing Sensor & 0 & $1620 \sim 1800$ & $x$ & $3980 \sim 4160$ & $x$ \\
\hline Ethanol & 50 & $1800 \sim 2160$ & 77.41 20.49 & $4160 \sim 5110$ & $102.87 \sim 17.17$ \\
\hline Air & 0 & $2160 \sim 2520$ & $20.49 \sim 79.77$ & $5110 \sim 6060$ & $17.17 \sim 100.62$ \\
\hline Replacing Sensor & 0 & $2520 \sim 2700$ & $x$ & $6060 \sim 6260$ & $x$ \\
\hline Ethanol & 50 & $2700 \sim 3060$ & $104.68 \sim 23.6$ & $6260 \sim 7210$ & $62.25 \sim 13.26$ \\
\hline Air & 0 & $3060 \sim 3600$ & $23.6 \sim 127.74$ & $7210 \sim 8200$ & $13.26 \sim 58.97$ \\
\hline
\end{tabular}

However, the proposed readout circuit updates $\mathrm{V}_{\mathrm{REF}}$ for every sensing measurement by tracking the initial value of $\mathrm{V}_{\mathrm{SO}}$. Sequentially, in Figure $5 \mathrm{c}, \mathrm{V}_{\mathrm{REF}}$ becomes $0.284 \mathrm{~V}, 0.65 \mathrm{~V}$, $0.572 \mathrm{~V}$, and $0.436 \mathrm{~V}$, and in Figure $6 \mathrm{c}, 0.706 \mathrm{~V}, 0.594 \mathrm{~V}, 0.443 \mathrm{~V}$, and $0.692 \mathrm{~V}$. As a result, regardless of the offset variation of gas sensors, the proposed readout circuit extracts only 
amount of change in the slow-responsive gas, while maintaining a uniform reactivity at the corresponding gas concentration.

In this study, we adopt the differential amplifier to extract the changed amount of the gas sensor, because it is advantageous to evaluate the performance of the proposed readout structure under various test environments. Since the amplifier performances are an important factor for determining signal-to-noise ratio (SNR) for readout structures, the readout structure with improved performances could be developed if further research is carried out on the amplifier circuitry.

\section{Measurement Results and Discussion}

Figure 7 shows the measurement environment for ethanol $\left(\mathrm{C}_{2} \mathrm{H}_{5} \mathrm{OH}\right)$ gas sensing that consists of a mass flow controller (MFC, Line Tech M3030V) for controlling the flow rate of $\mathrm{C}_{2} \mathrm{H}_{5} \mathrm{OH}$ gas and fresh air, a commercially available gas sensor array (the Figaro TGS2600), an evaluation board with the proposed readout circuit, and a measurement software program. The flow rate was controlled to $400 \mathrm{sccm}$ for $\mathrm{C}_{2} \mathrm{H}_{5} \mathrm{OH}$ gas and $300 \mathrm{sccm}$ for fresh air through the MFC. In addition, the injection time of $\mathrm{C}_{2} \mathrm{H}_{5} \mathrm{OH}$ gas and fresh gas was set to be from a minimum of $300 \mathrm{~s}$ to a maximum of $900 \mathrm{~s}$. Because the target ethanol gas is continuously injected into the sensor array under the MFC, there is no attenuation of the target gas concentration.

In this work, as shown in Figure 8a, the Figaro TGS2600 sensor was used for sensing $\mathrm{C}_{2} \mathrm{H}_{5} \mathrm{OH}$ gas at a concentration of $\sim 50 \mathrm{ppm}$ while considering its technical specifications. All gas-sensing measurements were conducted at normal temperature $\left(20 \pm 2{ }^{\circ} \mathrm{C}\right)$ and relative humidity is R.H. $65 \pm 5 \%$ in fresh air. Figure $8 \mathrm{~b}$ shows the evaluation board assembly that consists of the readout circuit board and commercial FPGA (XEM-7305 [22]) board. The readout circuit board has two gas-sensing channels to compare the performance of the conventional and proposed readout circuit structure. For further evaluation in various measurement environments, the control signals and operating timing are generated through the external FPGA mounted on the evaluation board. To verify compatibility and operation in terms of commercialization, in this work, the proposed readout structure was implemented on a PCB board using general-purpose discrete elements of differential amplifier, ADC, and DAC, to verify its effectiveness in various commercial gas-sensing systems. Thus, to enhance power efficiency, $1 \mathrm{kSPS}$ ADC/DAC was used where the ADC runs once every $100 \mathrm{~Hz}$ frequency for sensing the changes in the gas sensor. In order to consider bit resolution, since the noise performance of commercial gas sensors (Figaro TGS2600) was not disclosed, a low-noise differential amplifier and 14-bit noise-guaranteed ADC/DAC were used so that the noise limitation would not occur in the differential amplifier and ADC/DAC. Then, we observed the signal output, including noises of the gas sensor itself. Here, we chose the differential amplifier with noise performance of $16 \mathrm{nV} / \sqrt{\mathrm{Hz}}$ at $1 \mathrm{MHz}$ bandwidth and the 14-bit ADC/DAC with input referred noise of $0.36 \mathrm{LSB}_{\mathrm{rms}}$ at the full reference scale of $2.5 \mathrm{~V}$. In addition, to consider noise bandwidth, an additional external capacitor was added to the output node of the differential amplifier, resulting in a reduction of the noise bandwidth to $\sim 10 \mathrm{kHz}$. This can be further optimized when the proposed readout structure is developed into an integrated circuit. The output codes from the proposed readout circuit were transmitted to the PC through the USB interface and processed using a custom MATLAB software program to display the real-time imaging on the screen. The capturing software program with various verification functions was customized using MATLAB programming.

Figure 9 shows the output waveforms of the prototype readout circuit in which the measured ADC outputs are expressed as an analog output voltage. The offset tracking operation of the prototype readout circuit was verified using a ramping test input so that the gas sensor signal would be constant, but its offset increased linearly due to changes in the ambient environment. Here, a ramp signal with the slope of $6.67 \mathrm{mV} / \mathrm{s}$ was used. Compared to the conventional readout circuit, as shown in Figure 9a, the proposed readout circuit extracts the amount of change in the test input based on the initial value updated at 
each periodic offset tracking time. In addition, as shown in Figure $9 b$, it can be operated non-periodically according to any changes in conditions. The allowable offset tracking range is limited by the output dynamic range of the reference generating DAC.
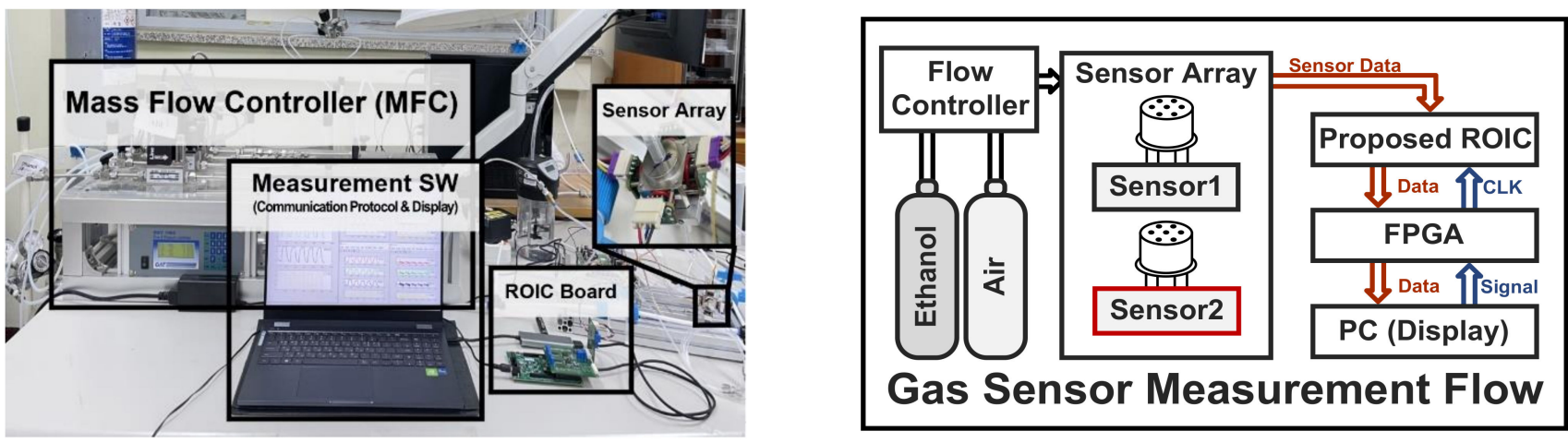

Figure 7. Gas-sensing measurement environment and gas sensor measurement flow.
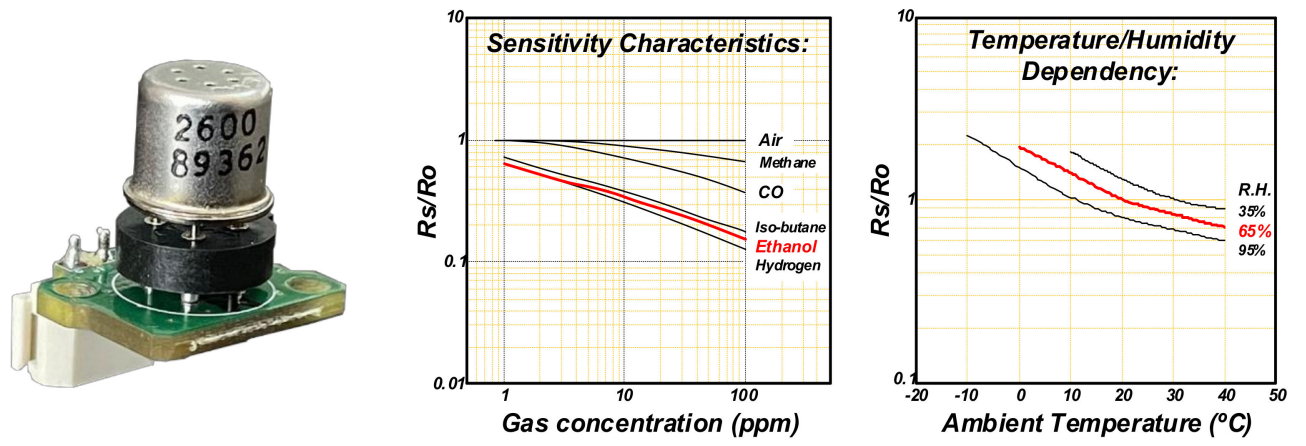

(a)
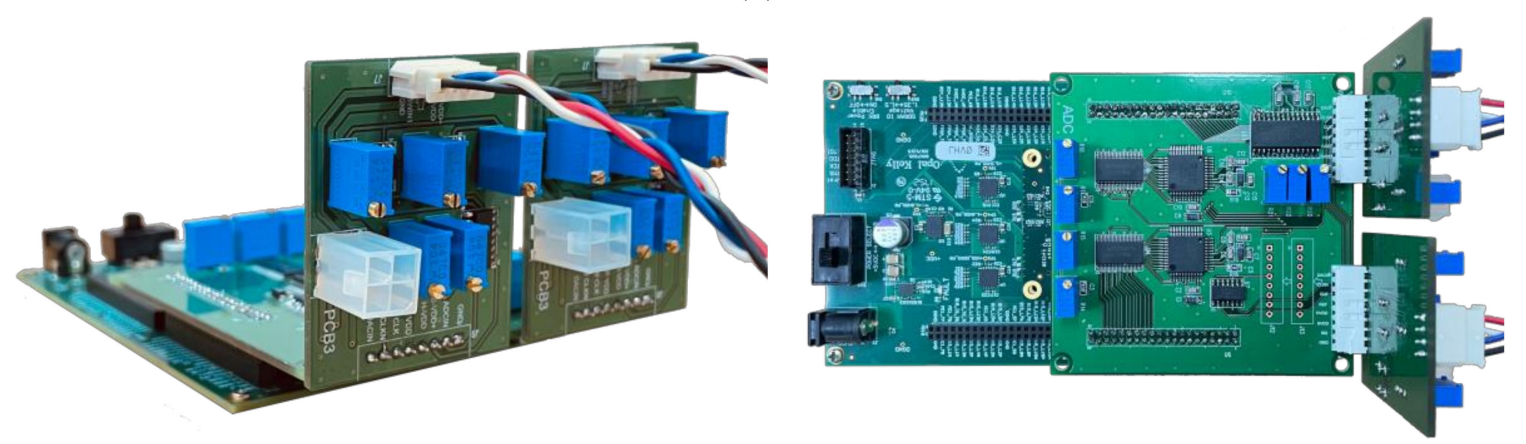

(b)

Figure 8. (a) Gas sensor board with Figaro TGS2600 and (b) the evaluation board with the proposed readout circuit.

Figure 10 shows the measurement results of the conventional readout circuit (representing the gray colored cells) and the proposed readout circuit (representing red colored) for two TGS2600 samples (Samples 1 and 2) with similar sensitivities but different initial values. To continuously compare the two sensors under the same test conditions, the gas sensor sample was replaced during the measurement (at $3800 \mathrm{~s}$ ). For a gas concentration of $50 \mathrm{ppm}$, from the conventional readout circuit, Sample 1 exhibits the output voltage of $3.31 \mathrm{~V}_{\mathrm{P} 1}$ at the initial value of $1.05 \mathrm{~V}_{\mathrm{OC} 1}$, whereas Sample 2 exhibits the output voltage of $3.44 \mathrm{~V}_{\mathrm{P} 2}$ at the initial value of $1.19 \mathrm{~V}_{\mathrm{OC} 2}$. Here, the average of the initial and output values of the sensing signal was used as a representative value. Owing to the difference in the initial value between the gas sensor samples, different sensor outputs are extracted even at the same gas concentration. From the perspective of a sensor system, this degrades the dis- 
crimination against each gas concentration changes, causing gas detection non-uniformity. On the contrary, in the case of the proposed readout circuit, the initial value of both samples is set to $0.67 \mathrm{~V}_{\mathrm{OC}}$, and based on this, Sample 1 and Sample 2 extract output voltages of $2.99 \mathrm{~V}_{\mathrm{P} 1}$ and $3.03 \mathrm{~V}_{\mathrm{P} 2}$, respectively. Considering the difference in sensitivity of the gas sensor sample itself, for the same gas concentration, these results are similar output values by minimizing the initial value difference.

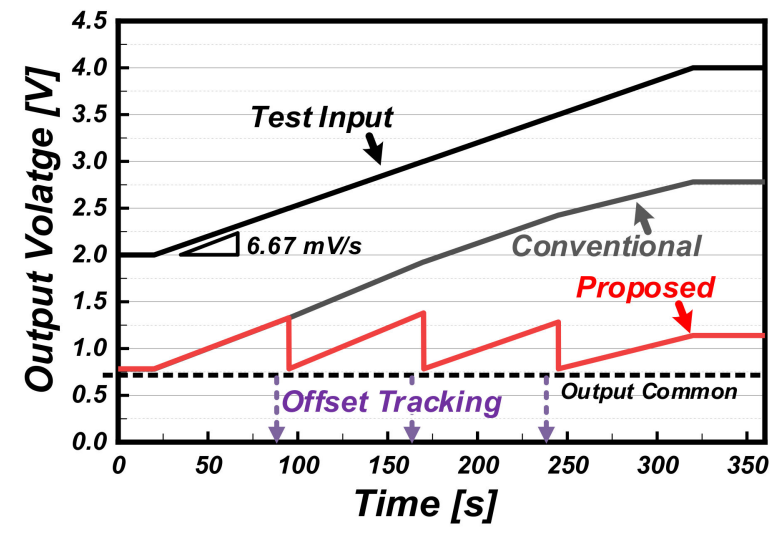

(a)

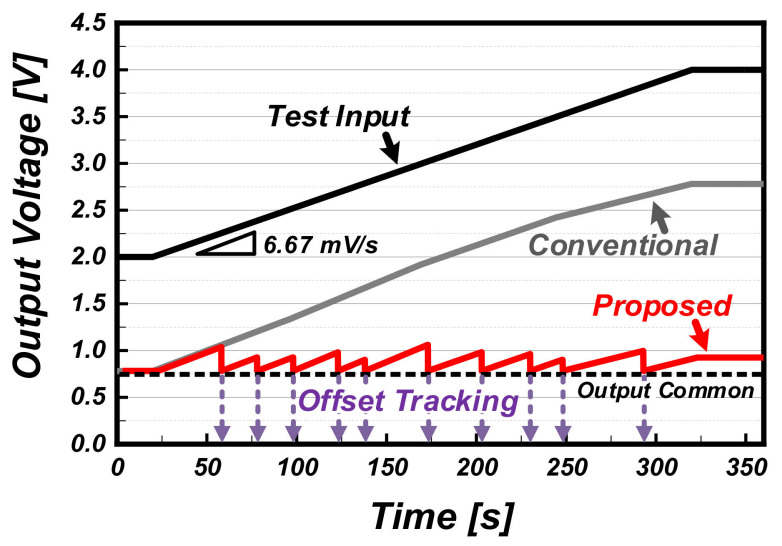

(b)

Figure 9. Measured output waveforms of conventional and proposed readout circuit under the condition of a linearly increasing sensor offset: (a) periodic offset tracking and (b) aperiodic offset tracking results.

Figure 11 shows the measurement results for various gas sensor samples (Samples 3,4 , and 5) with various sensitivities and initial values. In general, gas sensors have different initial values and different sensitivities for each gas sensor, so it is difficult to extract the uniform output value corresponding to an accurate gas concentration without a complicated post-processing process. However, the proposed readout circuit minimizes the difference in the initial value for each gas sensor, and it is effective even in compensating the difference in sensitivity for each gas sensor. Note that, for sensitivity compensation, the initial value of the gas sensor should be set to a constant value. Tables 2 and 3 list information on the measured sensor resistance per measurement time used in Figures 10 and 11 , respectively.

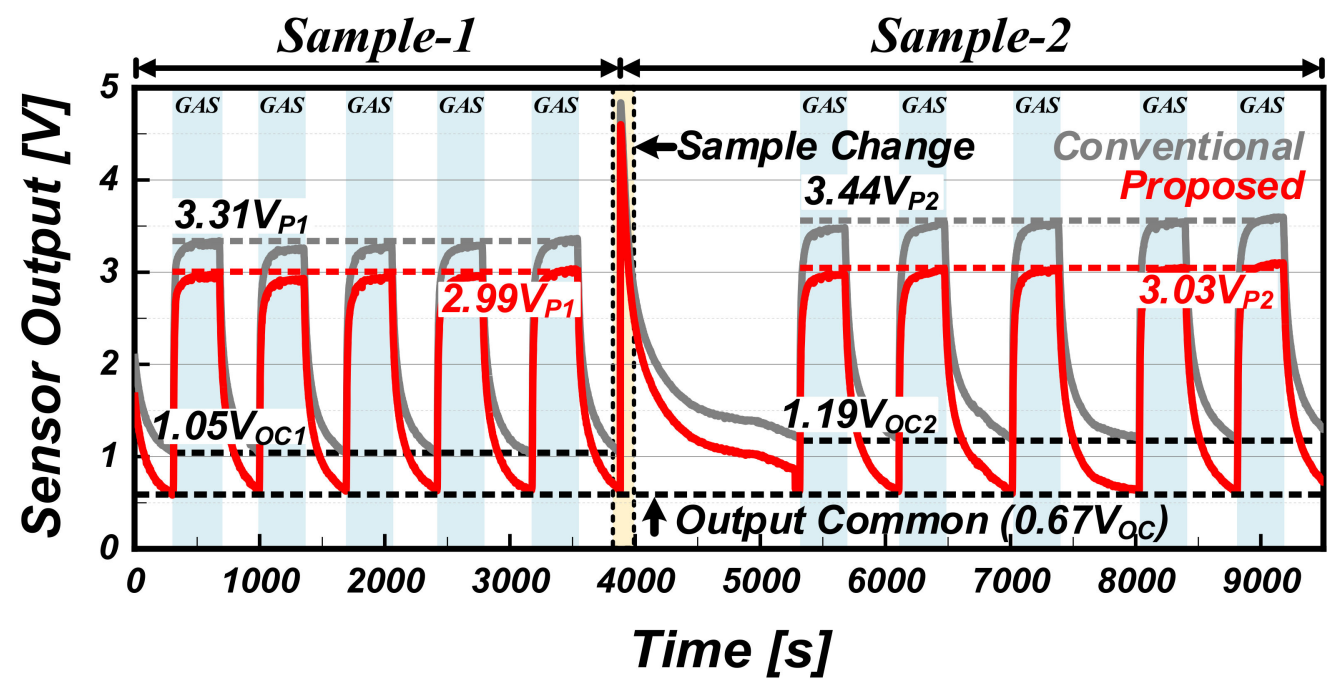

Figure 10. Measurement results of the conventional and proposed readout circuit for two commercial gas sensors. 


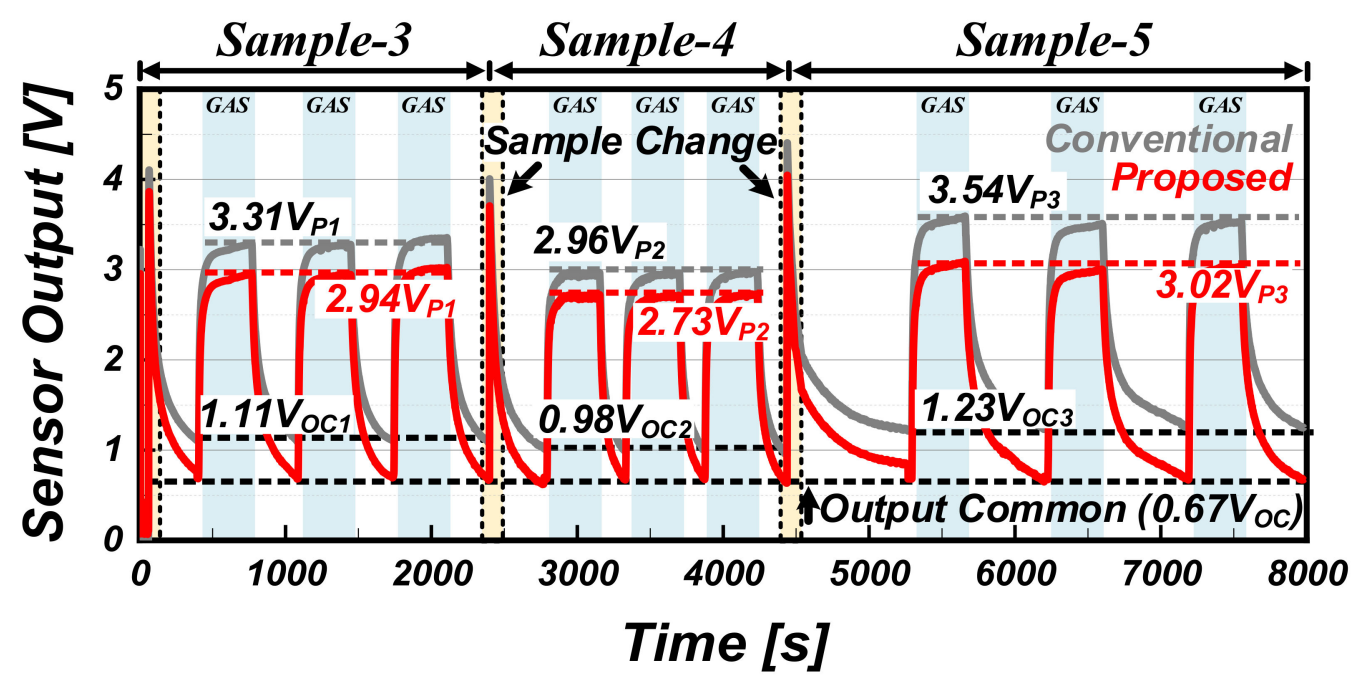

Figure 11. Measurement results for various gas sensor samples with various sensitivities and initial values.

It is meaningful to discuss how to accurately extract the signals of the gas sensors without the proposed offset tracking technique. Once the signals of the gas sensors are converted into digital information, a reference digital value should be defined for the digital signal to be meaningful information. However, considering the sensitivity of the gas sensor to the surrounding environment, it is not easy to determine a specific reference value that covers all measurement situations. As an example of digital processing, the output change from the gas sensor is recognized as a sensing signal by classifying digital signals through pattern recognition and defining minimum and maximum values. If this digital algorithm is implemented as digital logic or through post-processing, it is disadvantageous in terms of price competitiveness due to increased area occupation and power consumption. However, the proposed readout structure is relatively cost-effective to extract the amount of change from the gas sensor because it does not require any complex digital circuitry.

Table 2. Information regarding the measured sensor resistance per measurement time in Figure 10.

\begin{tabular}{|c|c|c|c|c|c|}
\hline \multirow[b]{2}{*}{ Input Condition } & \multirow{2}{*}{$\begin{array}{c}\text { Ethanol } \\
\text { Concentration } \\
\text { [ppm] }\end{array}$} & \multicolumn{2}{|c|}{ Sensor 1} & \multicolumn{2}{|c|}{ Sensor 2} \\
\hline & & $\begin{array}{c}\text { Measurement } \\
\text { Time [s] }\end{array}$ & $\begin{array}{c}\text { Mean Sensor } \\
\text { Resistance }[k \Omega]\end{array}$ & $\begin{array}{c}\text { Measurement } \\
\text { Time [s] }\end{array}$ & $\begin{array}{c}\text { Mean Sensor } \\
\text { Resistance }[\mathrm{k} \Omega]\end{array}$ \\
\hline Ethanol & 50 & $310 \sim 670$ & $109.4 \sim 15.31$ & $5310 \sim 5670$ & $93.96 \sim 13.06$ \\
\hline Air & 0 & $670 \sim 1000$ & $15.31 \sim 110.18$ & $5670 \sim 6110$ & $13.06 \sim 95$ \\
\hline Ethanol & 0 & $1000 \sim 1360$ & $110.18 \sim 16.04$ & $6110 \sim 6470$ & $95 \sim 12.5$ \\
\hline Air & 50 & $1360 \sim 1690$ & $16.04 \sim 110.58$ & $6470 \sim 7010$ & $12.5 \sim 95.52$ \\
\hline Ethanol & 0 & $1690 \sim 2050$ & $110.58 \sim 16.32$ & $7010 \sim 7370$ & 95.52 12.54 \\
\hline Air & 0 & $2050 \sim 2420$ & $16.32 \sim 108.88$ & $7370 \sim 8030$ & $12.54 \sim 92.54$ \\
\hline Ethanol & 50 & $2420 \sim 2780$ & $108.88 \sim 15.59$ & $8030 \sim 8390$ & $92.54 \sim 12.38$ \\
\hline Air & 0 & $2780 \sim 3180$ & $15.59 \sim 111.11$ & $8390 \sim 8810$ & $12.38 \sim 94.79$ \\
\hline Ethanol & 50 & $3180 \sim 3540$ & 111.11 14.77 & $8810 \sim 9170$ & $94.79 \sim 11.84$ \\
\hline Air & 0 & $3540 \sim 3880$ & $14.77 \sim 115.91$ & $9170 \sim 9700$ & $11.84 \sim 102.97$ \\
\hline Replacing Sensor & $X$ & $3880 \sim 5310$ & $x$ & $x$ & $X$ \\
\hline
\end{tabular}


Table 3. Information regarding the measured sensor resistance per measurement time in Figure 11.

\begin{tabular}{|c|c|c|c|c|c|c|c|}
\hline \multirow[b]{2}{*}{ Input Condition } & \multirow{2}{*}{$\begin{array}{c}\text { Ethanol } \\
\text { Concentration } \\
\text { [ppm] }\end{array}$} & \multicolumn{2}{|c|}{ Sensor 3} & \multicolumn{2}{|c|}{ Sensor 4} & \multicolumn{2}{|c|}{ Sensor 5} \\
\hline & & $\begin{array}{l}\text { Measurement } \\
\text { Time [s] }\end{array}$ & $\begin{array}{c}\text { Mean Sensor } \\
\text { Resistance }[k \Omega]\end{array}$ & $\begin{array}{l}\text { Measurement } \\
\text { Time [s] }\end{array}$ & $\begin{array}{c}\text { Mean Sensor } \\
\text { Resistance }[k \Omega]\end{array}$ & $\begin{array}{l}\text { Measurement } \\
\text { Time [s] }\end{array}$ & $\begin{array}{c}\text { Mean Sensor } \\
\text { Resistance }[k \Omega]\end{array}$ \\
\hline Ethanol & 50 & $410 \sim 770$ & 104.89 15.81 & $2790 \sim 3150$ & $120.6 \sim 20.74$ & $5300 \sim 5660$ & $93.45 \sim 12.24$ \\
\hline Air & 0 & $770 \sim 1090$ & $15.81 \sim 105.74$ & $3150 \sim 3340$ & $20.74 \sim 121.36$ & $5660 \sim 6240$ & $12.24 \sim 89.8$ \\
\hline Ethanol & 50 & $1090 \sim 1450$ & $105.74 \sim 16.21$ & $3340 \sim 3700$ & $121.36 \sim 20.65$ & $6240 \sim 6600$ & $89.8 \sim 12.78$ \\
\hline Air & 0 & $1450 \sim 1740$ & $16.21 \sim 104.77$ & $3700 \sim 3870$ & $20.65 \sim 120.75$ & $6240 \sim 7190$ & $12.78 \sim 89.6$ \\
\hline Ethanol & 50 & $1740 \sim 2100$ & $104.77 \sim 15.28$ & $3870 \sim 4230$ & $120.75 \sim 20.47$ & $7190 \sim 7550$ & $89.6 \sim 12.46$ \\
\hline Air & 0 & $2100 \sim 2400$ & $15.28 \sim 107.99$ & $4230 \sim 4440$ & $20.47 \sim 125.11$ & $7550 \sim 8000$ & $12.46 \sim 89.71$ \\
\hline Replacing Sensor & $x$ & $2400 \sim 2790$ & $X$ & $4440 \sim 5300$ & $X$ & $X$ & $X$ \\
\hline
\end{tabular}

\section{Conclusions}

This paper presents a commercially viable readout circuit structure targeting slow response gas sensors. In this paper, the optimized readout circuit structure for slow-reacting gas signals was verified in the simulation. It was then implemented on a PCB board to verify its effectiveness in various commercial gas-sensing systems. While maintaining the optimal operating condition of the gas sensor, regardless of the initial value of the gas sensor, the proposed readout circuit can effectively extract a uniform gas-sensing output for each gas concentration. In addition, the proposed design does not require large capacitors to extract the measurements, which will make the IC design more cost-effective and flexible. Although this study did not develop an integrated circuit (IC), the principle was proven using discrete components and the design could be the basis for developing an IC.

Author Contributions: This work was realized by the collaboration of all the authors. Conceptualization, D.-Y.L. and H.-J.K.; methodology, D.-Y.L. and H.-J.K.; software, D.-Y.L. and J.-B.Y.; validation, D.-Y.L. and J.-B.Y.; formal analysis, H.-G.B. and H.-J.K.; investigation, D.-Y.L., J.-B.Y. and H.-G.B.; resources, H.-G.B. and H.-J.K.; data curation, D.-Y.L. and J.-B.Y.; writing-original draft preparation, H.-J.K.; writing-review and editing, H.-G.B. and H.-J.K.; visualization, D.-Y.L.; supervision, H.-G.B. and H.-J.K.; project administration, H.-J.K.; funding acquisition, H.-G.B. All authors have read and agreed to the published version of the manuscript.

Funding: This research was supported by the National Research Foundation of Korea (NRF) grant funded by the Korea government (MSIT) (No. 2020R1I1A3074020) and the Korea Environmental Industry \& Technology Institute (2020002700011). This study was supported by a 2021 Research Grant from Kangwon National University.

Institutional Review Board Statement: Not applicable.

Informed Consent Statement: Not applicable.

Data Availability Statement: Not applicable.

Acknowledgments: The authors would like to thank IDEC for the EDA software support.

Conflicts of Interest: The authors declare no conflict of interest.

\section{References}

1. Itoh, T.; Miwa, T.; Tsuruta, A.; Akamatsu, T.; Izu, N.; Shin, W.; Park, J.; Hida, T.; Eda, T.; Setoguchi, Y. Development of an exhaled breath monitoring system with semiconductive gas sensors, a gas condenser unit, and gas chromatograph columns. Sensors 2016, 16, 1891. [CrossRef] [PubMed]

2. Choi, S.; Park, K.; Lee, S.; Lim, Y.; Oh, B.; Chae, H.Y.; Park, C.S.; Shin, H.; Kim, J.J. A Three-step resolution-reconfigurable hazardous multi-gas sensor interface for wireless air-quality monitoring applications. Sensors 2018, 18, 761. [CrossRef] [PubMed]

3. Yi, W.Y.; Leung, K.S.; Leung, Y.; Meng, M.L.; Mak, T. Modular sensor system (MSS) for urban air pollution monitoring. In Proceedings of the 2016 IEEE SENSORS, Orlando, FL, USA, 30 October-3 November 2016; pp. 1-3.

4. Zampolli, S.; Elmi, I.; Ahmed, F.; Passini, M.; Cardinali, G.C.; Nicoletti, S.; Dori, L. An electronic nose based on solid state sensor arrays for low-cost indoor air quality monitoring applications. Sens. Actuators B Chem. 2004, 101, 39-46. [CrossRef]

5. Cai, Y.; Gao, H.; Tong, B.; Li, M.; Arsad, N.; Li, Y. Measurement of trace ethane using a mid-IR LED. In Proceedings of the 2016 IEEE 6th International Conference on Photonics (ICP), Kuching, Malaysia, 14-16 March 2016; pp. 1-3. 
6. Wan, H.; Yin, H.; Lin, L.; Zeng, X.; Mason, A.J. Miniaturized planar room temperature ionic liquid electrochemical gas sensor for rapid multiple gas pollutants monitoring. Sens. Actuators B Chem. 2018, 255, 638-646. [CrossRef] [PubMed]

7. Manchukutty, S.; Vasa, N.J.; Agarwal, V.; Chandapillai, J. Dual photoionization source-based differential mobility sensor for trace gas detection in human breath. IEEE Sens. J. 2015, 15, 4899-4904. [CrossRef]

8. Drobek, M.; Kim, J.H.; Bechelany, M.; Vallicari, C.; Julbe, A.; Kim, S.S. MOF-based membrane encapsulated ZnO nanowires for enhanced gas sensor selectivity. ACS Appl. Mater. Interfaces 2016, 8, 8323-8328. [CrossRef] [PubMed]

9. Furst, L.; Feliciano, M.; Frare, L.; Igrejas, G. A portable device for methane measurement using a low-cost semiconductor sensor: Development, calibration and environmental applications. Sensors 2021, 21, 7456. [CrossRef] [PubMed]

10. Dey, A. Semiconductor metal oxide gas sensors: A review. Mater. Sci. Eng. B 2018, 229, 206-217. [CrossRef]

11. Choi, S.; Park, C.S.; Chae, H.Y.; Oh, B.; Lee, J.; Kwon, Y.M.; Baik, J.M.; Shin, H.; Kim, J.J. A wide dynamic range multi-sensor ROIC for portable environmental monitoring systems with two-step self-optimization schemes. IEEE Trans. Circuits Syst. I: Regul. Pap. 2021, 68, 2432-2443. [CrossRef]

12. Baranov, A.; Spirjakin, D.; Akbari, S.; Somov, A. Optimization of power consumption for gas sensor nodes: A survey. Sens. Actuators A Phys. 2015, 233, 279-289. [CrossRef]

13. Krebs, P.; Grisel, A. A low power integrated catalytic gas sensor. Sens. Actuators B Chem. 1993, 13, 155-158. [CrossRef]

14. Somov, A.; Karpov, E.F.; Karpova, E.; Suchkov, A.; Mironov, S.; Karelin, A.; Baranov, A.; Spirjakin, D. Compact low power wireless gas sensor node with thermo compensation for ubiquitous deployment. IEEE Trans. Ind. Inform. 2015, 11, 1660-1670. [CrossRef]

15. Hoon, K.; Won-Sup, C.; Hee-Jun, K.; Sang-Hee, S. A resistance deviation-to-pulsewidth converter for resistive sensors. IEEE Trans. Instrum. Meas. 2009, 58, 397-400. [CrossRef]

16. Kim, S.W.; Eom, W.J.; Choi, J.; Kim, J.J. Dual-mode wide-range linear CMOS interface circuit for resistive sensors. Electron. Lett. 2014, 50, 1575-1577. [CrossRef]

17. Park, K.; Choi, S.; Chae, H.Y.; Park, C.S.; Lee, S.; Lim, Y.; Shin, H.; Kim, J.J. An energy-efficient multimode multichannel gas-sensor system with learning-based optimization and self-calibration schemes. IEEE Trans. Ind. Electron. 2020, 67, 2402-2410. [CrossRef]

18. Choi, M.; Gu, J.; Blaauw, D.; Sylvester, D. Wide input range 1.7 $\mu \mathrm{W} 1.2 \mathrm{kS} / \mathrm{s}$ resistive sensor interface circuit with 1 cycle/sample logarithmic sub-ranging. In Proceedings of the 2015 Symposium on VLSI Circuits (VLSI Circuits), Kyoto, Japan, 17-19 June 2015; pp. C330-C331.

19. Ha, H.; Suh, Y.; Lee, S.; Park, H.; Sim, J. A 0.5V, 11.3- $\mu \mathrm{W}, 1-\mathrm{kS} / \mathrm{s}$ resistive sensor interface circuit with correlated double sampling. In Proceedings of the IEEE 2012 Custom Integrated Circuits Conference, San Jose, CA, USA, 9-12 September 2012; pp. 1-4.

20. Figaro Product Information for TGS2600. Available online: https://www.figarosensor.com/product/docs/tgs2600_ productinformation\%28fusa\%29_rev05.pdf (accessed on 20 December 2021).

21. Figaro Technical Information for TGS2600. Available online: https://www.figarosensor.com/product/data/tgs2600_technical_ infomation\%28fusa\%29_rev03.pdf (accessed on 20 December 2021).

22. Xem7305 User's Manual. Available online: https:/ / docs.opalkelly.com/xem7305 (accessed on 20 December 2021). 